\title{
ON PARTIAL DIFFERENTIAL AND DIFFERENCE EQUATIONS WITH SYMMETRIES DEPENDING ON ARBITRARY FUNCTIONS
}

\author{
Giorgio Gubbiotti, Decio Levi*, Christian Scimiterna \\ Dipartimento di Matematica e Fisica, Università degli Studi Roma Tre, e Sezione INFN di Roma Tre, Via della \\ Vasca Navale 84, 00146 Roma (Italy) \\ * corresponding author: decio.levi@roma3.infn.it
}

\begin{abstract}
In this note we present some ideas on when Lie symmetries, both point and generalized, can depend on arbitrary functions. We show a few examples, both in partial differential and partial difference equations where this happens. Moreover we show that the infinitesimal generators of generalized symmetries depending on arbitrary functions, both for continuous and discrete equations, effectively play the role of master symmetries.
\end{abstract}

KEYWORDS: partial differential equation; partial difference equation; Lie symmetries.

\section{INTRODUCTION}

In a seminal work of two century ago Medolaghi [27], following Sophus Lie results on ordinary differential equations [24, 25], proposed the following work program for Partial Differential Equations (PDE's):

(1.) Determine all different kinds of infinite groups of point transformations in 3 variables.

(2.) For each of the obtained groups determine the invariant second order equations.

In the framework of this research Medolaghi got, among other equations, the Liouville equation

$$
u_{x t}(x, t)=e^{u(x, t)} .
$$

The symmetry algebra of the Liouville equation 1.1 is given by the vector fields

$$
X(f(x))=f(x) \partial_{x}-f_{x}(x) \partial_{u}, \quad Y(g(t))=g(t) \partial_{t}-g_{t}(t) \partial_{u},
$$

where $f(x)$ and $g(t)$ are arbitrary smooth functions of their argument and by $f_{x}(x)$ and $g_{t}(t)$ we mean their first derivatives. The commutation relations of the vector fields $[1.2]$ are

$$
[X(f), X(\tilde{f})]=X\left(f \tilde{f}_{x}-\tilde{f} f_{x}\right), \quad[Y(g), Y(\tilde{g})]=Y\left(g \tilde{g}_{t}-\tilde{g} g_{t}\right), \quad[X(f), Y(g)]=0 .
$$

The algebra 1.2 , 1.3 is isomorphic to the direct sum of two Virasoro algebras.

We can find also $S$-integrable equations [5], i.e., equations integrable by the Spectral transform, which have an infinite dimensional group of point symmetries. Classical examples are the $(2+1)$-dimensional KadomtsevPetviashvili equation [7, 8, 29, the $(2+1)$-dimensional Davey-Stewartson equation [6], the $(2+1)$-dimensional Toda [22], the three wave interaction in three dimensions [26].

In the Example 5.7 of [28] one finds the point and generalized symmetries for the nonlinear first order wave equation

$$
u_{t}=u u_{x}, \quad u=u(x, t) .
$$

In evolutionary form they are given by the characteristic

$$
Q=u_{x} F\left(x+t u, u, t+\frac{1}{u_{x}}, \frac{u_{x x}}{u_{x}^{3}}\right),
$$

where the function $F$ is an arbitrary function of its arguments. In particular the last term corresponds to generalized symmetries as it depends of the second derivative of the field. For the symmetries of hydrodynamictype partial differential equations see [11].

Results by Shabat and Zhiber [37] show that also the Liouville equation, being Darboux integrable, has such kind of symmetries:

$$
Q=\left(D_{x}+u_{x}\right) F\left(w, w_{x}, \ldots, w_{k_{1} x}\right)+\left(D_{t}+u_{t}\right) G\left(\bar{w}, \bar{w}_{t}, \ldots, \bar{w}_{k_{2} t}\right),
$$


where the operators $D_{x}+u_{x}$ and $D_{t}+u_{t}$ are the Laplace operators, $k_{1}$ and $k_{2}$ are two positive integer numbers, $F$ and $G$ are two arbitrary functions of their arguments, $D_{x}$ and $D_{t}$ stands for the total derivative with respect to the $x$ and $t$ variables and $w=u_{x x}-\frac{1}{2} u_{x}^{2}$ and $\bar{w}=u_{t t}-\frac{1}{2} u_{t}^{2}$ are the lowest order integrals of the Liouville equation in the $x$ and $t$ direction. Here and in the following by $w_{n x}$ we mean the $n$ times derivative of the function $w(x, t)$ with respect to $x$.

So the nonlinear wave equation (1.4) as well as the Liouville equation (1.1) admit generalized symmetries depending on arbitrary functions. Then Medolaghi program could be extended to the case of generalized symmetries. We here present some preliminary ideas on a possible solution of this program.

In the following Section we will present some results on the construction of PDE's presenting generalized symmetries depending on arbitrary functions and relate them to Darboux integrable equations. Then in Section 3 we present the counterpart of the previous results in the discrete case. At the end we present few conclusive remarks and conjectures.

\section{Factorizable difFerential operators, Darboux integrable equations AND SYMMETRIES DEPENDING ON ARBITRARY FUNCTIONS.}

The result 1.5 can be in principle generalized to any differential equation of the first order as the equation for the symmetries, be them point or generalized ones, can be solved on the characteristics as it was in the case of the Hopf equation 1.4. Let consider, with no loss of generality, the example of a general first order autonomous equation in two independent variables,

$$
u_{t}=f\left(u, u_{x}\right), \quad u=u(x, t),
$$

where $f$ is an arbitrary function of its arguments. The symmetries are given by their characteristics $Q(x, t, u$, $\left.u_{x}, \ldots, u_{k x}\right)$ and their determining equations are given by

$$
D_{t} Q-\left.\left[\frac{\partial f}{\partial u} Q+\frac{\partial f}{\partial u_{x}} D_{x} Q\right]\right|_{u_{t}=f}=0 .
$$

Equation 2.2 is a first order differential equation for $Q$ which can be solved on the characteristics and whose solutions provide $k+2$ symmetry variables. Then, as in the case of $(1.5)$, the symmetries of (2.1) are given by arbitrary functions of the $k+2$ symmetry variables. So any first order PDE, linear or nonlinear, will have generically point and generalized symmetries depending on arbitrary functions of the symmetry variables.

We can easily show an interesting consequence of the existence of generalized symmetries depending on arbitrary functions. For the sake of concreteness we consider the symmetries of (1.4). Let us consider the subcase of (1.5) when

$$
Q_{f}=u_{x}\left[f_{1}(x+t u)+f_{2}\left(t+\frac{1}{u_{x}}\right)+f_{3}\left(\frac{u_{x x}}{u_{x}^{3}}\right)\right],
$$

i.e., the Hopf equation 1.4 has a symmetry generator of the form:

$$
\hat{X}_{f}=Q_{f} \partial_{u}
$$

with the functions $f_{i}, i=1,2,3$ analytic in their argument. When $f_{2}$ and $f_{3}$ are zero then 2.4 is the infinitesimal generator of point symmetries depending on an arbitrary function $f_{1}$; when $f_{1}$ and $f_{3}$ are zero then (2.4) is the infinitesimal generator of contact symmetries depending on an arbitrary function $f_{2}$; when $f_{1}$ and $f_{2}$ are zero then (2.4) is the infinitesimal generator of generalized symmetries depending on an arbitrary function $f_{3}$. If we take two of such generators, $\hat{X}_{f}$ and $\hat{X}_{g}$, where $f$ and $g$ are two different functions of the same argument, what can we say of their commutator? It has been proved by Bäcklund [17] that as soon as the characteristic $Q$ depends on derivatives of order higher than the first one, the symmetry group is infinite. The last symmetry group which can be finite is the one of the contact symmetries, if no arbitrary function is present. As was shown in the case of the Liouville equation the presence of a symmetry generator of point symmetries depending on an arbitrary function provide an infinite dimensional Lie algebra of point symmetries.

Let us carry out the the commutation of $\hat{X}_{f}$ and $\hat{X}_{g}$, for $f$ and $g$ equal to $f_{1}$ we get:

$$
\left[\hat{X}_{f_{1}}, \hat{X}_{g_{1}}\right]=\hat{X}_{f_{1} g_{1}^{\prime}-f_{1}^{\prime} g_{1}},
$$

equal to $f_{2}$ we get:

$$
\left[\hat{X}_{f_{2}}, \hat{X}_{g_{2}}\right]=0
$$


and equal to $f_{3}$ we get:

$$
\left[\hat{X}_{f_{3}}, \hat{X}_{g_{3}}\right]=\left[f_{3}^{\prime \prime} g_{3}^{\prime}-f_{3}^{\prime} g_{3}^{\prime \prime}\right] \frac{\left(u_{x x x} u_{x}-3 u_{x x}^{2}\right)^{2}}{u_{x}^{9}} \partial_{u}
$$

The algebra 2.5 turn out to be a Virasoro algebra of Lie point symmetries. The infinitesimal generators of the contact symmetries 2.6 commute but the infinitesimal generator depending on an arbitrary function of the generalized symmetry take the form of a master symmetry as the commutator of two such symmetries provide a symmetry of higher order (2.7).

The existence of arbitrary functions of generalized symmetries is not limited to the case of first order differential equations. It can easily extended to higher order PDE's when the differential operator which define the equation is factorizable. Few authors [18, 34] showed through the Laplace cascade method that factorizable linear PDE's are Darboux integrable if the cascade terminates. We can show that a factorizable PDE admits as a subclass of symmetries the symmetries of its first order PDE. As an example let us consider the case of second order factorizable PDE's. Let us consider the second order autonomous partial differential equation for one dependent variable $u$ in the two independent variables $x$ and $t$

$$
E_{1}=u_{t t}+\left[g\left(u, u_{x}\right)-f\left(u, u_{x}\right)_{u_{x}}\right] u_{x t}-f\left(u, u_{x}\right)_{u_{x}} g\left(u, u_{x}\right) u_{x x}-f\left(u, u_{x}\right)_{u}\left[u_{t}-g\left(u, u_{x}\right) u_{x}\right]=0,
$$

where $f$ and $g$ are two arbitrary functions of their arguments. Defining the first order autonomous PDE for $u=u(x, t)$,

$$
E_{0}=u_{t}-f\left(u, u_{x}\right)=0
$$

(2.8) is factorizable as:

$$
E_{1}=\left[\partial_{t}+g\left(u, u_{x}\right) \partial_{x}\right] E_{0}=0 .
$$

The symmetries of $E_{0}$ are given by the infinitesimal generator

$$
\hat{X}=Q_{0}\left(x, t, u, u_{x}, u_{t}, u_{x x}, \ldots\right) \partial_{u}
$$

and the determining equation is written as

$$
D_{t} Q_{0}-f\left(u, u_{x}\right)_{u} Q_{0}-\left.f\left(u, u_{x}\right)_{u_{x}} D_{x} Q_{0}\right|_{E_{0}=0}=0
$$

where $D_{t} Q_{0}$ and $D_{x} Q_{0}$ are the coefficient of the prolongation of $\hat{X}$ with respect to $u_{t}$ and $u_{x}$. The determining equation for the symmetries of $E_{1}=0$ of infinitesimal generator

$$
\hat{Y}=Q_{1}\left(x, t, u, u_{x}, u_{t}, u_{x x}, \ldots\right) \partial_{u}
$$

is given by

$$
\left.\operatorname{pr} \hat{Y} E_{1}\right|_{E_{1}=0}=0
$$

The explicit expression of (2.14) is long as it involves the application of the second prolongation of $\hat{Y}$. So we do not write it down here. By direct calculation it is easy to show that 2.14 is satisfied by the solution of 2.12. This shows that the symmetries of the second order autonomous factorizable PDE $E_{1}=0$ contain those of $E_{0}=0$. So $E_{1}=0$ can have arbitrary function dependent generalized symmetries as $E_{0}=0$ does. This proof can be extended to PDE's of any order and of any number of variables. The relation of this factorization to Laplace cascade method and Darboux integrability is still to be understood.

\section{Discrete EQUATIONS With GENERALIZED SYMMETRIES DEPENDING ON AN ARBITRARY FUNCTION.}

Partial Difference Equations (P $\Delta \mathrm{E}$ 's) can have symmetries depending on arbitrary functions. The presence of arbitrary functions of point symmetries has been shown to appear, as in the continuous case [3, 11, 17, 31, when the $\mathrm{P} \Delta \mathrm{E}$ is linearizable [21]. Here we show on some examples that one can find P $\Delta \mathrm{E}$ 's which have arbitrary functions of generalized symmetries. When they appear the system is usually linearizable but a complete theory in this case is absent. We have some results on Darboux integrable discrete equations [2, 9, 10, 32, 33, 35] which correspond to P $\Delta \mathrm{E}$ 's which have two distinct conserved quantities in the two different directions of the discrete plane. The complete classification of Darboux integrable equations on the lattice is absent and only 
some simple classes are worked out in the references mentioned above. Darboux integrable equations turn out to be linearizable but the other way around may not be true.

Here in the following we presents three linearizable P $\Delta \mathrm{E}$ 's [13, 14] which have arbitrary functions of generalized symmetries. Then, in a subsection, we present the case of the completely discrete Liouville equations. The first two equations are quad graph equations which do not possess the tetrahedron property. They turn out to be linearazable and have generalized symmetries of any order [14. Then we consider an equation of the Boll classification, ${ }_{t} H_{1}^{(\varepsilon)}$, which is non autonomous, linearizable and has three-point generalized symmetries [4, 13]. The three equations are:

(1.) The first equation belongs to the classification of compatible equations around the cube with no tetrahedron property presented by Hietarinta in [14]:

$$
v_{m+1, n} v_{m, n+1}+v_{m, n} v_{m+1, n+1}=0 .
$$

The three-point symmetries in the $m$-direction of $\sqrt{3.1}$ are given by the characteristic

$$
Q_{m, n}=v_{m, n} F\left((-1)^{n} \frac{v_{m+1, n}}{v_{m, n}},(-1)^{n} \frac{v_{m, n}}{v_{m-1, n}}\right) .
$$

As $(3.1)$ is symmetric in the exchange of $n$ and $m$ the generalized symmetry $(3.2)$ is valid also in the direction $n$ with the role of $n$ and $m$ interchanged.

We can easily find two conserved quantities

$$
W_{1}=(-1)^{n} \frac{v_{m+1, n}}{v_{m, n}}, \quad W_{2}=(-1)^{m} \frac{v_{m, n+1}}{v_{m, n}}
$$

such that

$$
\left(T_{2}-1\right) W_{1}=0, \quad T_{1} h_{m, n}=h_{m+1, n}, \quad\left(T_{1}-1\right) W_{2}=0, \quad T_{2} h_{m, n}=h_{m, n+1} .
$$

Then $(3.1)$ is Darboux integrable equation and $\sqrt{3.2}$ is just the three-point subcase of a general characteristic in the $m$ direction

$$
Q_{m, n}=v_{m, n} F\left((-1)^{n} T_{1}^{-N} \frac{v_{m+1, n}}{v_{m, n}}, \ldots,(-1)^{n} T_{1}^{M} \frac{v_{m+1, n}}{v_{m, n}}\right)
$$

with $N$ and $M$ two positive arbitrary integers such that the number of points involved in the symmetry is given by $N+M+2$. A similar characteristic exists also in the $n$ direction with the role of $n$ and $m$ interchanged.

Equation (3.1) is linearizable in three ways:

- By the point transformation $v_{0,0} \doteq e^{x_{0,0}}$, so that $x_{0,0}=\log v_{0,0}$ (without loss of generality, log can be always taken to stand for the principal value of the complex logarithm), is transformed into the linear equation $x_{0,0}-x_{1,0}-x_{0,1}+x_{1,1}=i \pi$.

- By the Hopf-Cole transformation $v_{1,0} / v_{0,0} \doteq w_{0,0}\left(v_{0,0} \neq 0\right)$ into the ordinary difference equation $w_{0,1}+w_{0,0}=0$.

- By the Hopf-Cole transformation $v_{0,1} / v_{0,0} \doteq t_{0,0}\left(v_{0,0} \neq 0\right)$ into the ordinary difference equation $t_{1,0}+t_{0,0}=0$.

(2.) A second equation which belongs to the classification of equations compatible around the cube with no tetrahedron property presented by Hietarinta in [14] is:

$$
\begin{aligned}
v_{m, n}+v_{m+1, n}+v_{m, n+1} & +v_{m+1, n+1}+v_{m+1, n} v_{m, n+1} v_{m+1, n+1} \\
& +v_{m, n}\left[v_{m+1, n} v_{m, n+1}+v_{m+1, n} v_{m+1, n+1}+v_{m, n+1} v_{m+1, n+1}\right]=0,
\end{aligned}
$$

whose three-point symmetries in the $m$-direction are given by

$$
Q_{m, n}=(-1)^{n}\left(1-v_{m, n}^{2}\right) K\left(\left(\frac{\left(1-v_{m, n}\right)\left(1-v_{m-1, n}\right)}{\left(1+v_{m, n}\right)\left(1+v_{m-1, n}\right)}\right)^{(-1)^{n}},\left(\frac{\left(1-v_{m, n}\right)\left(1-v_{m+1, n}\right)}{\left(1+v_{m, n}\right)\left(1+v_{m+1, n}\right)}\right)^{(-1)^{n}}\right) .
$$

Equation (3.6) admits a fourfold discrete symmetry given by

$$
v_{0,0} \rightarrow v_{0,0}^{(\varepsilon)} \doteq \frac{(1+\varepsilon) v_{0,0}+1-\varepsilon}{(1-\varepsilon) v_{0,0}+1+\varepsilon},
$$


where $\varepsilon$ is one of the four quartic roots of unity, $\varepsilon=( \pm i, \pm 1)$. Equation $[3.6)$ is symmetric in the exchange of $n$ and $m$ and consequently the generalized symmetry (3.7) is valid also in the direction $n$ interchanging the role of $n$ and $m$. It is not contained in the lists of Darboux integrable discrete equations [9] and [32], however we can prove that it is Darboux integrable as it admits the following integrals which satisfy (3.4):

$$
W_{1}=\left(\frac{\left(1-v_{m, n}\right)\left(1-v_{m+1, n}\right)}{\left(1+v_{m, n}\right)\left(1+v_{m+1, n}\right)}\right)^{(-1)^{n}}, \quad W_{2}=\left(\frac{\left(1-v_{m, n}\right)\left(1-v_{m, n+1}\right)}{\left(1+v_{m, n}\right)\left(1+v_{m, n+1}\right)}\right)^{(-1)^{m}} .
$$

The symmetry characteristic (3.7) is nothing but the reduction to three-point of a general case depending on $N+M+2$ points with $N$ and $M$ arbitrary positive integers,

$$
\begin{array}{r}
Q_{m, n}=(-1)^{n}\left(1-v_{m, n}^{2}\right) K\left(T_{1}^{-N}\left(\frac{\left(1-v_{m, n}\right)\left(1-v_{m-1, n}\right)}{\left(1+v_{m, n}\right)\left(1+v_{m-1, n}\right)}\right)^{(-1)^{n}}\right. \\
\left.\ldots, T_{1}^{M}\left(\frac{\left(1-v_{m, n}\right)\left(1-v_{m-1, n}\right)}{\left(1+v_{m, n}\right)\left(1+v_{m-1, n}\right)}\right)^{(-1)^{n}}\right) .
\end{array}
$$

A similar characteristic exists also in the $n$ direction with the role of $n$ and $m$ interchanged.

Equation 3.6$)$ is linearizable by the point transformation:

$$
v_{m, n} \doteq \frac{1+e^{x_{m, n}}}{1-e^{x_{m, n}}}
$$

into the linear equation

$$
x_{m, n}+x_{m+1, n}+x_{m, n+1}+x_{m+1, n+1}=2 i z \pi,
$$

where $z=-1,0,1,2$. The indeterminacy of the inhomogeneous term of 3.12 takes into account the fourfold discrete symmetry (3.8) of (3.6). It is worth while to notice that inverting (3.11) we get $x_{0,0}=\log \frac{v_{0,0}-1}{v_{0,0}+1}$, where without loss of generality, the function log can always be taken as the principal value of the complex logarithm [30]. Other two linearizations, similar to those showed for (3.1), are also present here.

(3.) ${ }_{t} H_{1}^{(\varepsilon)}$ is:

$$
\left(x_{m, n}-x_{m+1, n}\right)\left(x_{m, n+1}-x_{m+1, n+1}\right)-\varepsilon^{2} \alpha_{2}\left(F_{n}^{(+)} x_{m, n+1} x_{m+1, n+1}+F_{n}^{(-)} x_{m, n} x_{m+1, n}\right)-\alpha_{2}=0 .
$$

where $F_{n}^{( \pm)}=\frac{1 \pm(-1)^{n}}{2}$ and $\varepsilon$ and $\alpha_{2}$ are arbitrary constants. Equation 3.13 is Darboux integrable, as we can find two integrals in the $m$ and $n$ directions which satisfy (3.4). The $m$-integral being given by

$$
W_{2} \doteq a_{2} F_{n}^{(+)} s+b_{2} F_{n}^{(-)}\left(T_{2}+1\right) u, \quad s \doteq \frac{x_{m, n+1}-x_{m, n-1}}{1+\varepsilon^{2} x_{m, n+1} x_{m, n-1}}, \quad u \doteq x_{m, n}-x_{m, n-1},
$$

where $a_{2}$ and $b_{2}$ are two arbitrary constants. The $n$ direction integral is different, as ${ }_{t} H_{1}^{(\varepsilon)}$ is not symmetric in the exchange of $n$ and $m$, and is given by

$$
W_{1} \doteq F_{n}^{(+)} \frac{\alpha_{2}}{v}+F_{n}^{(-)} t, \quad t \doteq \frac{x_{m, n}-x_{m-1, n}}{1+\varepsilon^{2} x_{m-1, n} x_{m, n}}, \quad v \doteq x_{m, n}-x_{m-1, n}
$$

Equations (3.14) and (3.15) are effectively four integrals as $a_{i}$ and $b_{i}, i=1,2$ are independent constants.

In 12 we constructed its three-point generalized symmetries along the direction $m$ :

$$
\begin{array}{r}
Q_{m, n}=F_{n}^{(+)}\left(\frac{\alpha_{2}\left(v^{2}+\varepsilon^{2} \alpha_{2}^{2}\right)}{(\bar{v}-v)(\bar{v}+v)} B_{m}\left(\frac{\alpha_{2}}{\bar{v}}\right)-\frac{\alpha_{2}\left(\bar{v}^{2}+\varepsilon^{2} \alpha_{2}^{2}\right)}{(\bar{v}-v)(\bar{v}+v)} B_{m-1}\left(\frac{\alpha_{2}}{v}\right)+\left(x_{m, n}-\frac{\left(\bar{v}^{2}+\varepsilon^{2} \alpha_{2}^{2}\right) v}{(\bar{v}-v)(\bar{v}+v)}\right) \omega+\gamma_{n}\right) \\
+F_{n}^{(-)}\left(\frac{\bar{t}^{2} t^{2}}{(\bar{t}-t)(\bar{t}+t)}\left(B_{m}(\bar{t})-B_{m-1}(t)\right)-\frac{\bar{t}^{2} t}{(\bar{t}-t)(\bar{t}+t)} \omega+\delta_{n}\right)\left(1+\varepsilon^{2} x_{m, n}^{2}\right)
\end{array}
$$

where $B_{m}(y), \gamma_{n}$ and $\delta_{n}$ are generic functions of their arguments, $\omega$ is an arbitrary constant and $\bar{v}=T_{1} v$ and $\bar{t}=T_{1} t$. Let us note that any free function and free parameter may eventually depend on $\alpha_{2}$ and $\varepsilon$. It is possible to demonstrate that, as long as $\varepsilon \neq 0$, no $n$-independent reduction of the above symmetry exists. If in 3.16 the functions $B_{m-1}$ appearing in it will depend on the variables $T_{1}^{p} W_{1}, \ldots, T_{1}^{p+N} W_{1}$ 
and consequently $B_{m}$ on $T_{1}^{p+1} W_{1}, \ldots, T_{1}^{p+1+N} W_{1}$, one obtains the generalized symmetries for (3.13) in the direction $m$ at all order.

The three-point generalized symmetry along the direction $n$ is different as the equation is not symmetric. It is:

$$
Q_{m, n}=F_{n}^{(+)}\left(B_{n}(s)+\kappa_{n}\right)+F_{n}^{(-)}\left(1+\varepsilon^{2} x_{m, n}^{2}\right)\left(C_{n}(\bar{u}+u)+\lambda_{n}\right),
$$

where $B_{n}(y)$ and $C_{n}(y)$ are arbitrary functions of their argument and of the lattice variable $n, \bar{u}=T_{2} u$ and $\kappa_{n}$ and $\lambda_{n}$ are arbitrary functions of the lattice variable $n$. Due to the complexity of the three-point generalized symmetries we are not able, in this case, to evince the Laplace operators and thus write the generalized symmetries depending on an arbitrary function for any number of points.

As in the case of PDE's presented in the previous Section also here the generalized symmetry depending on an arbitrary function play the role of a master symmetry. Let us consider, as an example, the commutation of two generalized symmetries of (3.1) of infinitesimal generators $\hat{X}_{F_{m}}=v_{m} F_{m} \partial_{v_{m}}$ and $\hat{X}_{G_{m}}=v_{m} G_{m} \partial_{v_{m}}$ characterized by the arbitrary functions $F_{m}=F\left((-1)^{n} \frac{v_{m+1}}{v_{m}}\right)$ and $G_{m}=G\left((-1)^{n} \frac{v_{m+1}}{v_{m}}\right)$ corresponding to (3.5) with $N=M=0$. We have:

$$
\left[\hat{X}_{F_{m}}, \hat{X}_{G_{m}}\right]=(-1)^{n} v_{m+1} H_{m} \partial_{v_{m}}
$$

where

$$
H_{m}=\left[\Delta F_{m} G_{m}^{\prime}-\Delta G_{m} F_{m}^{\prime}\right]
$$

In $3.19, F_{m}^{\prime}$ means the derivative of $F_{m}$ with respect to its argument and the operator $\Delta$ is such that $\Delta F_{m}=F_{m+1}-F_{m}$. The function $H_{m}$ depends on more points than the functions $F_{m}$ and $G_{m}$ and so the generator $\hat{X}_{F_{m}}$ plays the role of a master symmetry.

\subsection{The Discrete Algebraic Liouville Equations}

Among the many different completely discrete Liouville equations which go in the continuous limit in the algebraic Liouville equation

$$
v v_{x y}-v_{x} v_{y}=v^{3}
$$

obtained from 1.1 by setting $v=e^{u}$, let's mention the following four:

- The Tzitzeica-Liouville equation, given in [1]:

$$
h_{m, n} h_{m+1, n+1}\left(h_{m+1, n}-1\right)\left(h_{m, n+1}-1\right)-\left(h_{m, n}-1\right)\left(h_{m+1, n+1}-1\right)=0 ;
$$

- The potential Hirota-Liouville equation, given in [15, 33]:

$$
v_{m, n} v_{m+1, n+1}-v_{m+1, n} v_{m, n+1}+1=0 ;
$$

- The Hirota-Liouville equation, given in [16, 33]:

$$
u_{m, n} u_{m+1, n+1}-\left(u_{m+1, n}-1\right)\left(u_{m, n+1}-1\right)=0 ;
$$

- The Adler-Startsev Liouville equation, given in [2]:

$$
t_{m, n} t_{m+1, n+1}\left(1+\frac{1}{t_{m+1, n}}\right)\left(1+\frac{1}{t_{m, n+1}}\right)-1=0 .
$$

These P $\Delta$ E's can be transformed one into the other by the following transformations:

$$
u_{m, n}=\frac{h_{m, n}}{h_{m, n}-1}, \quad h_{m, n} \neq 1, \quad u_{m, n}=v_{m+1, n} v_{m, n+1}, \quad u_{m, n}=-\frac{1}{t_{m, n}} .
$$

The generalized symmetries of (3.21) along the direction $m$ are given for all $p \in \mathcal{Z}$ and $N \in \mathcal{N}_{0}$ by

$$
\frac{\mathrm{d} t_{m, n}}{\mathrm{~d} \varepsilon}=-\left(1+t_{m, n}\right)\left(T_{1}-1\right)\left(1+\frac{t_{m, n}\left(1+t_{m-1, n}\right)}{t_{m-1, n}}\right)^{-1} t_{m, n} f\left(T_{1}^{p} W_{1}, \ldots, T_{1}^{p+N} W_{1}\right)
$$

where $\varepsilon$ is the group parameter, $f(x, y, \ldots, z)$ is an arbitrary function of its arguments and $W_{1}$ is the Darboux integral of the Liouville equation (3.21) along the direction $n$, given in [2] by

$$
W_{1}=\left(1+\frac{t_{m, n}\left(1+t_{m-1, n}\right)}{t_{m-1, n}}\right)\left(1+\frac{t_{m, n}}{t_{m+1, n}\left(1+t_{m, n}\right)}\right),
$$


satisfying (3.4). Equation $(3.23)$ is the equivalent of formula $(1.6)$ introduced by Zhiber and Shabat for the continuous Liouville equation.

Choosing $p=-1$ and $N=1$ in $(3.23)$ we obtain the most general generalized symmetry depending at most on the five points $t_{m-2, n}, t_{m-1, n}, t_{m, n}, t_{m+1, n}$ and $t_{m+2, n}$

$$
\frac{\mathrm{d} t_{m, n}}{\mathrm{~d} \varepsilon}=-\left(1+t_{m, n}\right)\left(T_{1}-1\right)\left(1+\frac{t_{m, n}\left(1+t_{m-1, n}\right)}{t_{m-1, n}}\right)^{-1} t_{m, n} g\left(T_{1}^{-1} W_{1}, W_{1}\right) .
$$

If $\partial_{x} g(x, y) \neq 0$ and $\partial_{y} g(x, y) \neq 0$, then we get a five-point symmetry; if $\partial_{x} g(x, y)=0$ and $\partial_{y} g(x, y) \neq 0$, then we get a four-point symmetry depending on the asymmetric set $t_{m-1, n}, t_{m, n}, t_{m+1, n}$ and $t_{m+2, n}$; if $\partial_{x} g(x, y) \neq 0$ and $\partial_{y} g(x, y)=0$, then we get a four-point symmetry depending on the asymmetric set $t_{m-2, n}, t_{m-1, n}, t_{m, n}$ and $t_{m+1, n}$; finally if $g=1$ we get the three-point symmetry

$$
\frac{\mathrm{d} t_{m, n}}{\mathrm{~d} \varepsilon}=-\left(1+t_{m, n}\right)\left(T_{1}-1\right)\left(1+\frac{t_{m, n}\left(1+t_{m-1, n}\right)}{t_{m-1, n}}\right)^{-1} t_{m, n}
$$

which doesn't depend on any arbitrary function. As it is evident from $(3.23)$ there is no way that we can get a Lie point symmetry in agreement with the results presented in [19, 20. The lowest possible symmetry is a generalized symmetry depending on three points 3.25 . Equation 3.21 is symmetric in the exchange of the $m$ and $n$ indices. So the symmetries in the $n$ directions are trivially given by $(3.23)$ with $T_{1}$ substituted by $T_{2}$ and the shifts in $m$ substituted by shifts in $n$.

\section{Conclusive Remarks}

In this note we presented a set of results partially distributed in a series of articles of different authors concerning the presence of symmetries depending on arbitrary functions both in the continuous and in the discrete setting. Few of the words associated to these systems are Darboux integrable systems, linearizable systems, factorizable differential operators but not all necessarily proved to be connected to the presence of symmetries depending on arbitrary functions. Darboux integrable systems in two independent variables are systems, studied primarily by G. Darboux, characterized by the presence of at least a couple of conserved quantities in the two independent variables. As far as it is known in the literature Darboux integrable systems are linearizable and the primary example is the Liouville equation (1.1) which has also arbitrary function dependent symmetries, both point and generalized [38].

Here we showed that generically first order PDE's have symmetries depending on arbitrary functions as the symmetry determining equations are characterized by just first order PDE's which can be solved on the characteristics in term of arbitrary functions of the symmetry variables. These same symmetries can be found in PDE's of higher order when the differential operator is factorized in the product of lower order ones which, at the bottom, is just a first order one. Tsarev in [34] correlate Darboux integrable systems with factorizable differential operators.

The situation is less clear in the discrete setting. The classification of Darboux integrable systems is not complete for P $\Delta$ E's even on the square graph. Few results [2, 35] are known on factorizable difference operators in the framework of Darboux integrable systems and symmetries. Nothing has been done up to now to characterize partial difference equations whose symmetries are written in term of arbitrary functions of symmetry variables. Moreover, let us notice that the generalized symmetries we obtain for Darboux integrable equations as (3.5), (3.10) and (3.23) do not define, in general, $S$-integrable differential difference equations. Indeed these symmetries do not always satisfy the necessary condition for the $S$-integrability, that the highest order shift in the lattice variable is the opposite of the lowest one [23, 36].

As a result of this note we can conjecture the following theorem:

Theorem 1. Necessary and sufficient conditions for a PDE and a P $\Delta E$ to have symmetries, possibly point but surely generalized, depending on arbitrary functions is that the system be Darboux integrable.

Some of the results presented here are new and never presented anywhere. Among them let us mention the structure of the Lie algebra of the symmetries of the Hopf equation, the fact that both for PDE's and $\mathrm{P} \Delta \mathrm{E}$ 's generalized symmetries characterized by arbitrary functions provide master symmetries. Moreover the calculations of the generalized symmetries, the Darboux integrals and the linearizing transformation for the two nonlinear quad graph equations introduced by Hietarinta are new here as well as the generalized symmetries for the completely discrete Liouville equation and the Darboux integrals for ${ }_{t} H_{1}^{(\varepsilon)}$.

\section{ACKNOWLEDGEMENTS}

CS and DL have been partly supported by the Italian Ministry of Education and Research, 2010 PRIN Continuous and discrete nonlinear integrable evolutions: from water waves to symplectic maps.

GG and DL are supported by INFN IS-CSN4 Mathematical Methods of Nonlinear Physics. 


\section{REFERENCES}

[1] V. E. Adler On a discrete analog of the Tzitzeica equation, preprint arXiv:1103.5139v1, March $26^{\text {th }}, 2011$.

[2] V. E. Adler and S. Ya. Startsev, Discrete analogues of the Liouville equation, Theor. Math. Phys., 121 (1999) 1484-1495, DOI:10.1007/BF02557219

[3] G. Bluman and S. Kumey, Symmetries and Differential Equations, Springer-Verlag, New York, 1989, DOI:10.1007/978-1-4757-4307-4

[4] R. Boll, Classification and Lagrangian structure od 3D consistent quad-equations, Ph. D. dissertation (2012).

[5] F. Calogero and W. Eckhaus, Nonlinear Evolution Equations, Rescalings, Model PDEs and their Integrability. I \& II. Inv. Probl. 3 (1987) 229-262, DOI:10.1088/0266-5611/3/2/008 \& 4 (1988) 11-33, DOI:10.1088/0266-5611/4/1/005

[6] B. Champagne and P. Winternitz, On the infinite-dimensional symmetry group of the Davey-Stewartson equantions, J. Math. Phys, 29 (1988) 1-8, DOI:10.1063/1.528173

[7] D. David, N. Kamran, D. Levi and P. Winternitz, Subalgebras of loop algebras and symmetries of the Kadomtsev-Petviashvili equation, Phys. rev. lett. 55 (1985) 2111-2113, DOI:10.1103/physrevlett.55.2111

[8] D. David, N. Kamran, D. Levi and P. Winternitz, Symmetry reduction for the Kadomtsev-Petviashvili equation using a loop algebra, J. Math. Phys. 27 (1986) 1225-1237, DOI:10.1063/1.527129

[9] R. N. Garifullin and R. I. Yamilov, Generalized symmetry classification of discrete equations of a class depending on twelve parameters, J. Phys. A: Math. Theor. 45 ( 2012$) 345205$ (23 pp.), DOI:10.1088/1751-8113/45/34/345205

[10] R. N. Garifullin, R. I. Yamilov, Examples Of Darboux Integrable Discrete Equations Possessing First Integrals Of An Arbitrarily High Minimal Order, Ufimsk. Mat. Zh., 4 (2012), 174-180.

[11] A. M. Grundland, M. B. Sheftel and P. Winternitz, Invariant solutions of hydrodynamic-type equations, J. Phys. A: Math. Gen. 33 (2000) 8193-8215, DOI:10.1088/0305-4470/33/46/304

[12] G. Gubbiotti, C. Scimiterna and D. Levi, Linearizability and fake Lax pair for a consistent around the cube nonlinear non-autonomous quad-graph equation, arXiv:1510.01527, submitted to Theor. Math. Phys. .

[13] G. Gubbiotti, C. Scimiterna and D. Levi, The non autonomous YdKN equation and generalized symmetries of Boll equations, arXiv:1510.07175, submitted to J. Phys. A.

[14] J. Hietarinta, Searching for CAC-maps, J. Nonlinear Math. Phys. 12 Suppl. 2 (2005) 223-230, DOI:10.2991/jnmp.2005.12.s2.16

[15] R. Hirota, Nonlinear partial difference equations. V. Nonlinear equations reducible to linear equations, J. Phys. Soc. Japan 46 (1979) 312-319, DOI:10.1143/jpsj.46.312

[16] R. Hirota, Discrete two-dimensional Toda molecule equation, J. Phys. Soc. Japan 56 (1987) 4285-4288, DOI:10.1143/jpsj.56.4285

[17] N. H. Ibragimov, Transformation Groups Applied to Mathematical Physics, Nauka, Moscow, 1983 (English translation by Reidel, Dordrecht, 1985), DOI:10.1007/978-94-009-5243-0

[18] M. Juráš and I. M. Anderson, Generalized Laplace Invariants and the Method of Darboux, Duke Math. J. 89 (1997) 351-375, DOI:10.1215/s0012-7094-97-08916-x

[19] D. Levi, L. Martina and P. Winternitz, Lie-point symmetries of the discrete Liouville equation, J. Phys. A: Math. Theor. 48 (2015) 025204 (18 pp.), DOI:10.1088/1751-8113/48/2/025204 arXiv:1407.4043

[20] D. Levi, L. Martina and P. Winternitz, Structure Preserving Discretizations of the Liouville Equation and their Numerical Tests, SIGMA 11 (2015) 080 (20 pp.), DOI:10.3842/sigma.2015.080 arXiv:1504.01953v2

[21] D. Levi and C. Scimiterna, Linearization through symmetries for discrete equations, J. Phys. A: Math. Theor. 46 (2013) 325204 (18 pp), DOI:10.1088/1751-8113/46/32/325204

[22] D. Levi and P. Winternitz, Continuous symmetries of discrete equations, Phys. Lett. A 152 (1991) 335-338, DOI:10.1016/0375-9601(91)90733-o

[23] D. Levi and R. I. Yamilov, Conditions for the existence of higher symmetries of evolutionary equations on the lattice, J. Math. Phys. 38 (1997) 6648-6674, DOI:10.1063/1.532230

[24] S. Lie, Über Gruppen von Transformationen, Gottinger Nachrichten 1874 (1874) 529-542.

[25] S. Lie, articles published mostly in the Norwegian Archive and reproduced in the essential part in: Allgemeine Theorie der partiellen Differentialgleichungen erster Ordnung Math. Ann. 9 (1875) 245-296, DOI:10.1007/bf01443377, Theorie der Transformationsgruppen I, Math. Ann. 16 (1880), 441-528, DOI:10.1007/bf01446218; Allgemeine Untersuchungen über Differentialgleichungen, die eine continuirliche, endliche Gruppe gestatten, Math. Ann. 25 (1885) 71-151, DOI:10.1007/bf01446421; Classification und Integration von gewöhnlichen Differentialgleichungen zwischen xy, die eine Gruppe von Transformationen gestatten, Math. Ann. 32 (1888) 213-281, DOI:10.1007/bf01444068

[26] L. Martina and P. Winternitz, Analysis and applications of the symmetry group of the multidimensional three wave resonant interaction problem, Ann. Physics 196 (1989) 231-277, DOI:10.1016/0003-4916(89)90178-4 
[27] P. Medolaghi, Classificazione delle equazioni alle derivate parziali del secondo ordine, che ammettono un gruppo infinito di trasformazioni puntuali, Ann. Mat. Pura Appl. 1 (1898) 229-263, DOI:10.1007/bf02419192

[28] P. J. Olver, Applications of Lie Groups to Differeial Equations, (second edition), Springer, New York, 1993, DOI:10.1007/978-1-4612-4350-2

[29] F. Schwarz, Symmetries of the Two-Dimensional Korteweg-deVries Equation, J. Phys. Soc. Jpn. 51 (1982) 2387-2389, DOI:10.1143/jpsj.51.2387

[30] C. Scimiterna and D. Levi, Classification of discrete equations linearizable by point transformation on a square lattice, Front. Math. China 8 (2013) 1067-1076, DOI:10.1007/s11464-013-0280-3

[31] M. B. Sheftel, Symmetry group analysis and invariant solutions of hydrodynamic-type systems, Int. J. Math. Math. Sci. 2004 (2004), 487-534, DOI:10.1155/s0161171204206147

[32] S. Ya. Startsev, Darboux integrable discrete equations possessing an autonomous first-order integral, J. Phys. A: Math. Theor. 47 (2014) 105204 (16pp), DOI:10.1088/1751-8113/47/10/105204

[33] S. Ya. Startsev, Non-Point Invertible Transformations and Integrability of Partial Difference Equations, SIGMA 10 (2014) 066 (13 pp.), DOI:10.3842/sigma.2014.066

[34] S. P. Tsarev, Factoring linear partial differential operators and the Darboux method for integrating nonlinear partial differential equations, Teoret. Mat. Fiz. 122 (2000), 144-160; English transl., Theoret. Math. Phys. 122 (2000), 121-133, DOI:10.1007/bf02551175

[35] V. L. Vereshchagin, Darboux-Integrable Discrete Systems, Theor. Math. Phys., 156 (2008) 1142-1153, DOI:10.1007/s11232-008-0084-x

[36] R. I. Yamilov, Symmetries as integrability criteria for differential difference equations, J. Phys. A: Math. Gen. 39 (2006) R541-R623, DOI:10.1088/0305-4470/39/45/r01

[37] A. V. Zhiber and A. B. Shabat, Klein-Gordon equations with a nontrivial group, Dokl. Akad. Nauk SSSR 247 (1979), 1103-1107; English transl., Soviet Phys. Dokl. 24 (1979), 607-609.

[38] A. V. Zhiber and V. V. Sokolov, Exactly integrable hyperbolic equations of Liouville type, Uspekhi Mat. Nauk 56 (2001) 63-106; English transl., Russian Math. Surveys 56 (2001) 61-101, DOI:10.1070/rm2001v056n01abeh000357 